\title{
Physiological and psychological responses to surgery and non-surgery days in orthopaedic surgeons: A case study
}

\author{
Sarantis Abatzoglou*1, Kevin Milne ${ }^{2}$, James L. Howard ${ }^{1}$, Emily Dobbin ${ }^{1}$, Filip Zekic ${ }^{1}$, Brent Lanting ${ }^{1}$ \\ ${ }^{1}$ Division of Orthopaedic Surgery, Department of Surgery, University Hospital, London Health Sciences Center, University of \\ Western Ontario, London, Ontario, Canada \\ ${ }^{2}$ Department of Kinesiology, Faculty of Human Kinetics, University of Windsor, Windsor, Ontario, Canada
}

Received: December 9, 2016

Accepted: February 8, 2017

Online Published: February 14, 2017

DOI: $10.5430 /$ css.v3n2p7

URL: http://dx.doi.org/10.5430/css.v3n2p7

\begin{abstract}
Objective: Performing surgical procedures can increase the physiological stress and mental strain experienced by practicing surgeons. This may affect negatively work capacity and quality of patient care by increasing the incidence of burnout, sleep disorders, fatigue and substance abuse. The purpose of this pilot case study was to track physiological and psychological responses to clinical duties in an expert arthroplasty orthopaedic surgeon.

Methods: Heart rate (HR), breathing rate, heart rate variability (HRV) and anxiety ratings were observed in an expert orthopaedic surgeon using a wearable Equivital EQO2 physiological monitor and the state-trait anxiety inventory (STAI), respectively. Data was recorded for 8 surgery, 1 clinic and 1 rest day. Physiological data was recorded for 6-8 hours/day.

Results: The surgeon spent significantly more time with elevated HRs on surgery days versus either clinic or rest days (10\% of the day over $100 \mathrm{bpm}$ ). This was not the case for respiratory rate. STAI responses were significantly greater pre versus post surgery and at baseline. Psychological and physiological markers of stress were elevated prior to each surgery regardless of surgery type and task. Notably, the HR never returned to baseline on surgery days.

Discussion: This study provides a baseline on which to compare procedural and trainee differences during surgical procedures. Expert surgeons may demonstrate elevated stress levels on days of surgery, as indicated by self-reported anxiety scores as well as physiologic measures. Understanding the stress and anxiety present may direct interventions that enable improved surgeon health, surgical performance, and an improved learning environment.
\end{abstract}

Key Words: Stress, Heart rate, Surgical training, Heart rate variability, Remote monitoring

\section{INTRODUCTION}

Any challenge, real or perceived, to physiological homeostasis causes stress and counter-regulatory responses. These responses are mutli-functional and may include molecular, physiological, cognitive and/or behavioral changes. Nonetheless, the body is always under a certain amount of stress as physiological homeostasis is maintained under changing internal and external environments. Elevated stress improves physical and mental performance in a variety of activities, however, this occurs only up to a point after which performance declines and health is negatively affected. ${ }^{[1]}$

The surgeon, surgical team, and surgical trainees are exposed

\footnotetext{
${ }^{*}$ Correspondence: Sarantis Abatzoglou; Email: sarantis.abatzoglou@mail.mcgill.ca; Address: Division of Orthopaedic Surgery, Department of Surgery, University Hospital, London Health Sciences Center, University of Western Ontario, London, Ontario, Canada.
} 
to physical and mental stress during surgery. The cumulative effect of this stress can affect not only tolerable work capacity by increasing the incidence of burnout, ${ }^{[2]}$ but may also result in the deterioration of the quality of patient care. ${ }^{[2]}$ The efficiency, efficacy, and the surgeon's skill levels may be negatively affected if the surgeon or other members of the operative team are experiencing elevated stress. ${ }^{[3]}$ Moreover, the learning environment may be deteriorated, impeding the surgical trainees' ability to acquire new skills. ${ }^{[4]}$

Unfortunately, medical and surgical training are not immune to excessive stress. A large study examined burnout in an internal medicine residency group, regardless of whether residents met the criteria for burnout or not. ${ }^{[5]}$ The participants in this study most often rated inadequate sleep, frequently working shifts longer than 24 hours and inadequate leisure time as major stresses. These stresses could result from or could cause higher incidences of physiological stress or potentiate experienced stresses. For example, acute sleep deprivation is associated with impaired autonomic regulation of the heart. ${ }^{[6]}$ Work stress, shift work, and length of shift are associated with reduced cardiovascular and mental health. ${ }^{[7,8]}$ Inadequate leisure time leads individuals to exercise less and have less family time. ${ }^{[9]}$ Moreover, residents exhibit poor dietary practices during residency, ${ }^{[10]}$ that would undoubtedly be a part of an increase in the likelihood of reduced health outcomes, poor performance, and reduced patient care. ${ }^{[11]}$ These events are not limited to residents, but may be common in practicing surgeons as well. For example, in a population of surgeons, burnout has been reported as high as $40 \%$ in a large ( $>7,000$ individuals) sample of American surgeons. ${ }^{[11]}$

The perception of fatigue tends to correlate well with actual physical fatigue. ${ }^{[12]}$ Moreover, physical stress is associated with increases in HR, breathing rate and body temperature. HR variability (HRV) has been demonstrated to be one of the best methods for autonomic stress assessment. ${ }^{[13]} \mathrm{HRV}$ can be calculated using frequency domain analysis of the beat-to-beat variation in HR. Within the frequency domain analysis, low frequency (LF) component is associated with sympathetic and vagal modulation of the heart while the high frequency (HF) component is representative of vagal modulation of the heart. Consequently, the ratio of low to high frequencies (LF/HF Ratio) is representative of the balance between sympathetic and vagal activity. A higher ratio represents a predominant sympathetic response, and therefore a period of higher stress while a lower ratio represents a predominantly parasympathetic response. Measuring HRV provides greater sensitivity than measuring HR alone, as an elevated HR alone may be due to physical exertion in the absence of other types of stress (e.g. anxiety). ${ }^{[13]}$ This type of observation has been used in sports training in order to identify stressors and optimize the athlete's training and overall performance ${ }^{[14]}$ However, few studies have analyzed actual physiological responses during surgical practice, ${ }^{[4,15-17]}$ and what factors may modify (i.e. attenuate) these responses. Consequently, the goal of this study was to track physiological and psychological responses to common hip and knee surgeries, as well as during clinic and rest days in an expert orthopaedic surgeon.

\section{MATERIAL AND METHODS}

Consent was obtained from an expert surgeon from a single institution, the participant was fully explained the methods to be used throughout the study. An independent research assistant, who is not one of the main investigators, collected all data. All procedures received institutional human research ethics clearance from the institutions involved in the study.

The participant completed the State-Trait Anxiety Questionnaire (state-trait anxiety inventory [STAI], Y1 and Y2) at the beginning of the study. The STAI is a validated tool used to measure the subjective level of state (i.e. how anxious a subject is in the present situation) and trait (i.e. the tendency to perceive a given situation as stressful) anxiety. ${ }^{[18]}$ It consists of 40 questions (20 question for state and 20 questions for trait anxiety) that measure self-reported scales of feelings of apprehension, tension, nervousness, and worry. Higher scores in this test indicate higher levels of anxiety as a stable trait (STAI-Y1) or acutely (STAI-Y2). The test was administered to the participant on day 1 of the study, which was a non-surgical day as well as at the commencement of the other 24-hour non-surgical work days in order to establish anxiety-stress levels experienced. On surgery work days, the participant was administered the STAI-Y2 (state) questionnaire at the beginning of the day prior to surgeries, immediately prior to each surgery and after individual surgeries were completed.

The participant was also instructed to wear an Equivital EQO2 HRV holter monitor (Vivonoetics, San Diego, California) for 6-8 hours on non-surgery workdays, on surgery workdays for the entirety of the respective workdays and for 6-8 hours during the rest day. Physiological data collected by the device included electrocardiogram (EKG), respiratory rate using strain gauge technology, infrared skin temperature. All data was sent electronically to the investigators for analysis of the data and calculation of HR and HRV using VivoSense $\mathbb{R}$ Software (Vivonoetics, San Diego, California). For each surgery for which data was collected, analysis was performed starting 10 minutes prior to the start of the surgery (Pre-operative period) up to 10 minutes following that procedure (Post-operative period). The results of the STAI ques- 
tionnaires allowed for comparisons with the data acquired via the HR monitor, to determine whether perceived stress correlates with physiological changes.

Given that we observed the data for a single individual, simple correlation analysis was made between the data collected from the questionnaire and the physiological responses noted with the monitoring.

\section{Results}

One expert orthopaedic surgeon specialized in adult hip and knee reconstruction with 3 years of practice working at a single teaching hospital facility was included in the study. Data was collected for a total of 10 days, including 8 surgery days, 1 clinic day and 1 "off"/rest day. A total of 22 surgeries were performed during that period, including fifteen total hip arthroplasty (THA) and seven total knee arthroplasty (TKA).

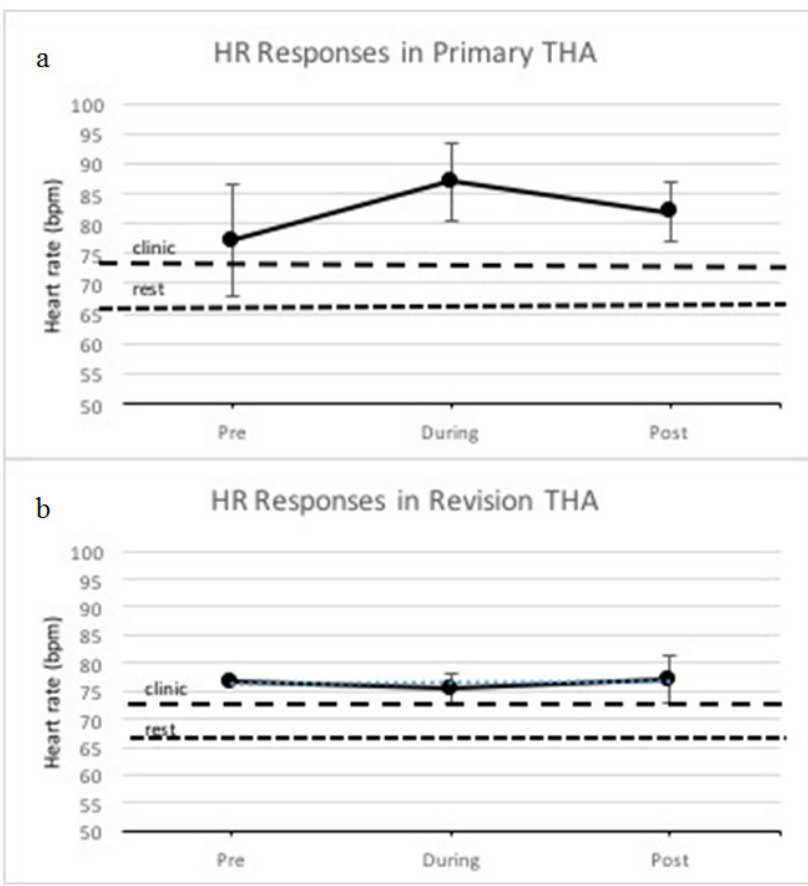

Figure 1. Mean heart rate recorded in (a) Primary THA (pTHA) and (b) Revision THA (rTHA) (OR data shown with solid line)

Mean HR was 66 beats per minute (bpm) during the rest day and $71 \mathrm{bpm}$ during clinic day. The HR during surgery days was elevated throughout the day regardless of the period measured (see Figures 1 and 2) HR was higher during the perioperative and intraoperative period performing THA compared to TKA. Before THA and TKA, the mean HR was $77 \mathrm{bpm}$ and $75 \mathrm{bpm}$ respectively. Mean HR increased to 87 bpm (SD $6.6 \mathrm{bpm}$ ) during THA and to $76.5 \mathrm{bpm}$ (SD 4.2 bpm) during TKA. The HR post-operatively never reached the pre-operative levels after THA (mean $82 \mathrm{bpm}$, SD $5.0 \mathrm{bpm}$ ), but did after TKA (mean 75 bpm, SD $2.4 \mathrm{bpm}$ ).

Skin temperature was similar during the rest day and clinic days. During surgery days, it was 1 degree higher than the other days throughout the day. No differences were noted when comparing between the different type of surgeries performed.

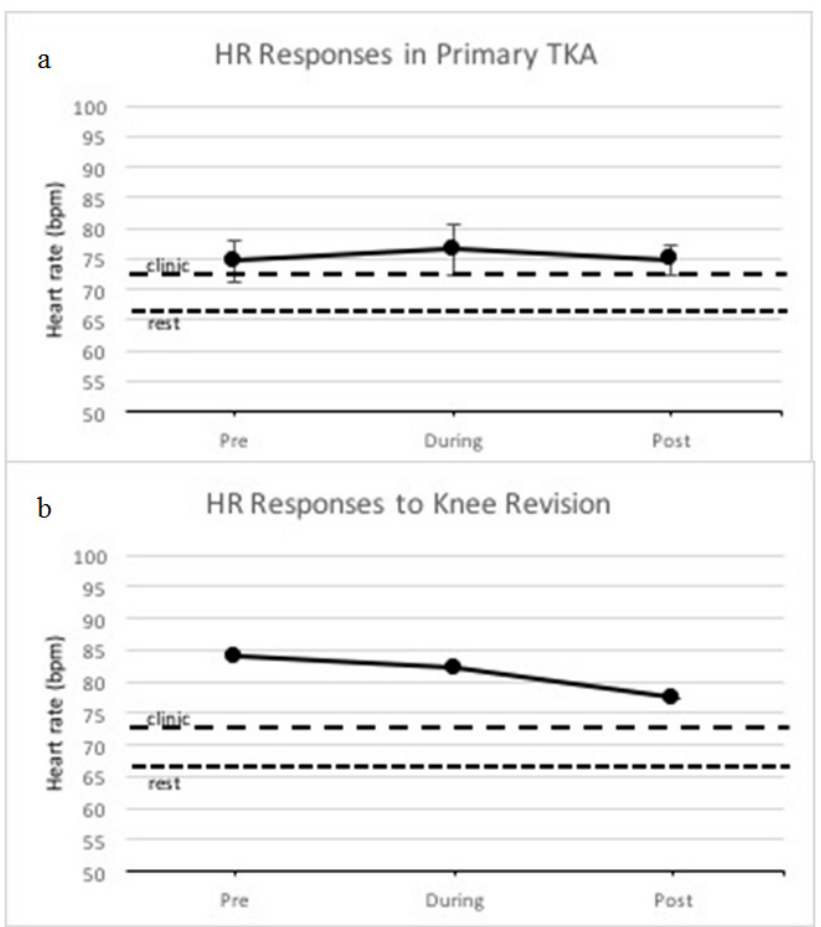

Figure 2. Mean heart rate recorded in (a) Primary TKA (pTKA) and (b) Revision TKA (rTKA) (OR data shown with solid line)

Respiratory rate was elevated on surgery days compared to the rest ( 17 breaths per minute) and clinic day ( 15.3 breaths per minutes). Respiratory rate was increased before starting any procedure (Mean THA 22.5 breaths per minute, SD 4.3 - Mean TKA 19.9 breaths per minute, SD 2.6). It remained elevated but at slightly lower levels during THA (Mean 20.2 breaths per minute, SD 2.2), however reached similar levels to rest days during TKA (Mean 17.4 breaths per minute, SD 2.8). In the immediate postoperative period, the respiratory rate remained at the same levels as intraoperative in both THA (Mean 21.5 breaths per min, SD 4.5) and TKA (Mean 17.9 breaths per min, SD 0.1).

HRV was measured as a ratio of LF/HF. Results are seen in Figures 3 and 4 . This ratio was slightly increased before any procedure, affected more before THA (mean LF/HF 4.39, $\mathrm{SD} 4.05$ ) than in TKA (mean LF/HF 2.20, SD 1.62). It was even higher during and after any procedure. During a THA, 
the $\mathrm{LF} / \mathrm{HF}$ reaches a mean of 7.30 (SD 3.75) and remains high after the procedure to a mean of 6.80 (SD 4.45). Similarly, during a TKA, the $\mathrm{LF} / \mathrm{HF}$ ratio increased to a lesser level than the other procedure reaching a mean 5.09 (SD 2.46) intraoperatively and mean of 4.46 (SD 2.90) after the procedure.

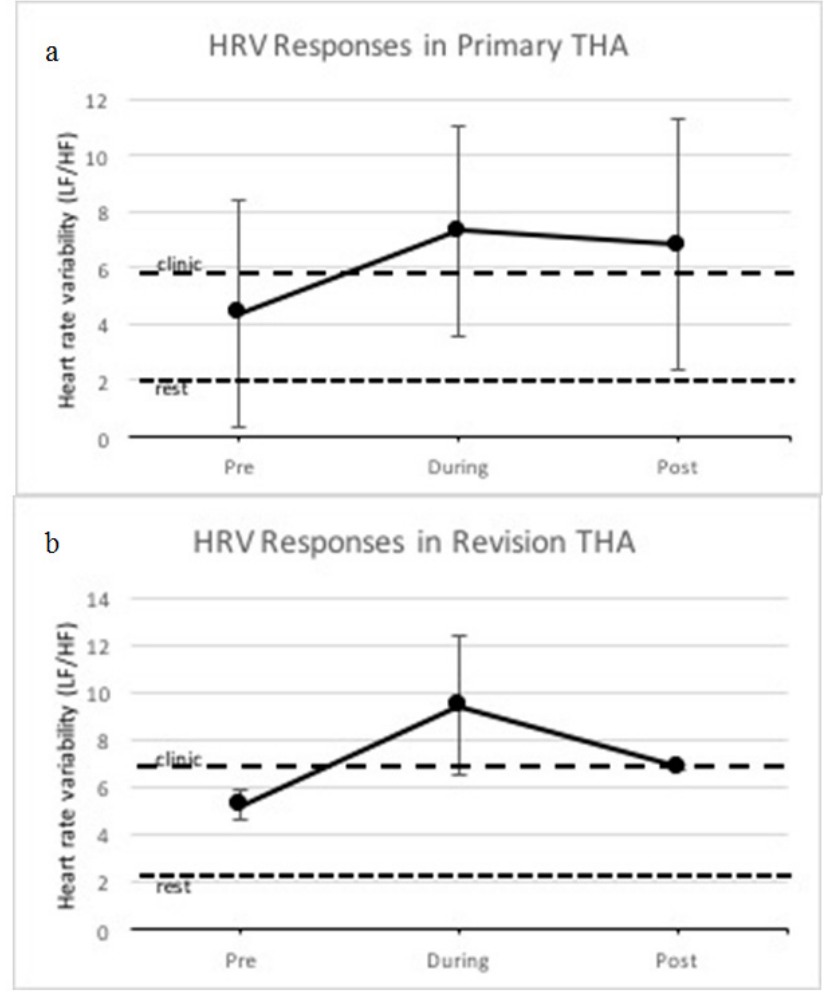

Figure 3. HRV recorded in (a) Primary THA and (b) Revision THA (OR data shown with solid line)

Evaluating the responses to the STAI questionnaire before and after any procedure, average score was consistently higher preoperatively than postoperatively. Before THA, the STAI questionnaire score has a mean of 32.9 compare to 22.1 after the procedure. When performing TKA, the STAI questionnaire score reaches a mean of 30.5 preoperatively compare to 20 postoperatively. There was no correlation noted between the STAI scores and physiological responses like HR $\left(R^{2}=0.15683\right)$ or HRV $\left(R^{2}=0.11333\right)$.

\section{Discussion}

The aim of this study was to track physiological and psychological responses to common hip and knee surgeries, as well as during clinic and "off" days in an expert orthopaedic surgeon. Similar pilot studies have been done in other surgical specialties. ${ }^{[18,19]}$ However, this study is the first involving common orthopaedic surgery procedures and physiological responses, since only questionnaire-based studies have been done in this field. ${ }^{[20]}$ Common orthopaedic surgical procedures are unique since they are much more physically demanding than other type of surgeries. The physicality of these surgeries adds to the physiological responses experienced by the surgeon to the stressors surrounding the surgery itself. This makes it even more important to use HRV as our measurement tool to perform such analysis.

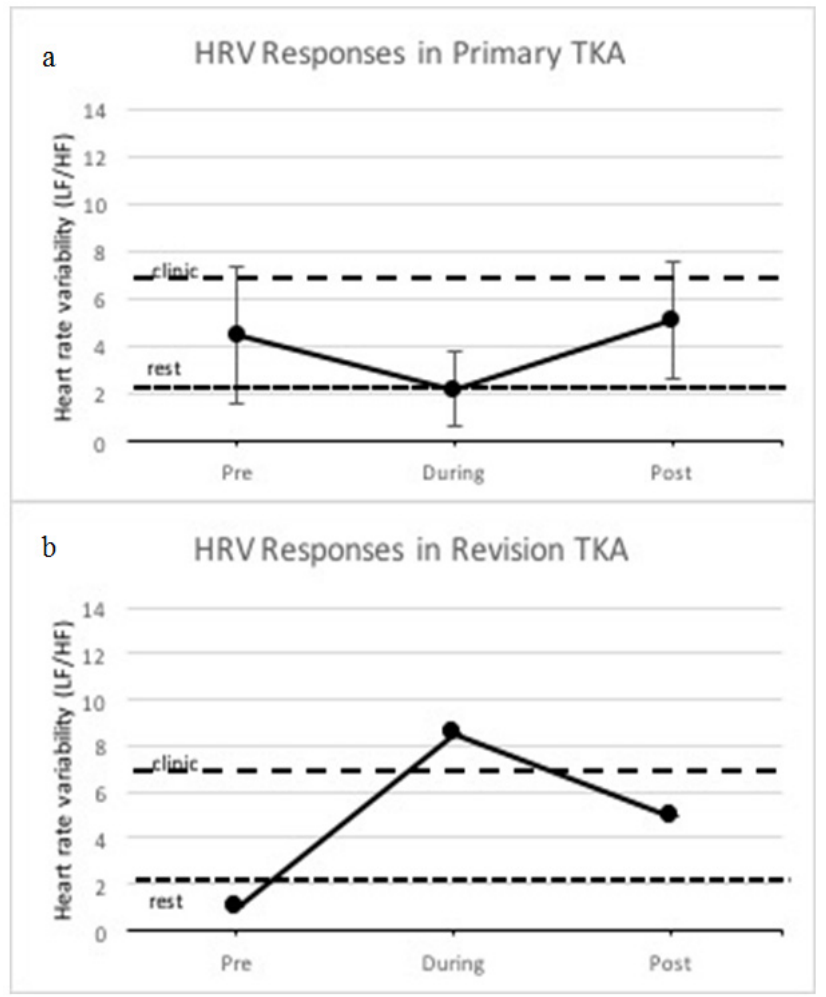

Figure 4. HRV recorded in (a) Primary TKA and (b) Revision TKA (OR data shown with solid line)

Physiological responses tended to be different between the type of procedures performed. Specifically, they were higher during THA compared to TKA. Multiple factors could potentially explain such results. First, this could be related to the increased physical stress experienced during the procedure. Second, the complexity of the procedure might differ between the THA and the TKA for the sample of operations for which data was collected. Third, the surgeon participating in this pilot study performed anterior approach THA at the time of data collection. The surgeon was the only surgeon performing the THA using this approach at our institution at that time. Therefore, the surgeon might have experienced higher stress levels during THA, as other members of the surgical team (nurses and trainees) were relatively inexperienced with this surgical technique. Future study with multiple surgeons performing a variety of surgical techniques will help to further explore these issues. 
Moreover, this study clearly shows that despite the minimal amount of physical activity happening in the immediate preoperative period, the physiological responses during that period are higher than rest days or clinic days. When combined with the increased scores in the STAI questionnaires during the same period, we understand that a surgeon has an increased level of stress during the surgery days. This relates probably to surgeon related psychological stresses of patient safety, self-imposed demands, and high achievement standards.

The STAI questionnaire has been previously used in the literature to evaluate stress levels in surgeons. ${ }^{[3,21]}$ However, no studies before correlated STAI scores with physiological responses. It is difficult to establish such correlation in our limited observations. However, we were able to observe a tendency towards increased physiological responses in the postoperative period in contrast to STAI scores that were similar to the rest day scores during that same postoperative timeframe. This could indicate that most of the physiological response observed in the postoperative period is related to physical strain recovery rather than response to stressors.

The limitations of this study are as follows. First, it is difficult to differentiate between physical and mental stress during surgery given the physical nature of the tasks and the inability to get subjective ratings of stress. Second, noise in the EKG tracing collected might have interfered with the results obtained. We anticipate this effect would be small as the Vivosense software uses automatic filters to diminish the effect of the noise on the collected data as much as possible. Third, the results obtained from the STAI questionnaires could have been biased since filling these questionnaires on multiple times during a same day could lead to acute repetition of data by the participant and, therefore, might influence the results obtained.

The results of this study show the feasibility in detecting physiological levels of stress in orthopaedic surgeons in their everyday work related activities. This study will be used as the basis to a larger scale study including multiple surgeons, trainees during those surgeries using different types of approaches and other orthopaedic procedures. As competency based training models are becoming increasingly implemented, evaluating different stressors surrounding the surgical training will eventually lead to improvements in the learning environment for the surgical trainees, and lead to more efficient and healthier training. Of particular importance will be correlating the timing of stress (i.e. at what surgical step) for surgeons and surgical trainees to understand if different interventions may be required at different times of a given procedure.

\section{Conclusions}

In conclusion, this pilot study demonstrates that an established surgeon experiences increased physiologic stress and psychologic stress on days that surgery is performed. Surgeons experience much lower stress and anxiety on leisure days and days spent in clinic with outpatients. It is notable that different levels of stress are encountered in different types of procedures. Further investigation to evaluate and identify stressors in the hospital setting, identify their effect on learning and practice, and to provide interventions to optimize the environment for both the surgeon as well as other members of the health care provider team and trainees is required.

\section{CONFlicts OF INTEREST Disclosure}

The authors declare they have no conflict of interest.

\section{REFERENCES}

[1] Nixon PG. The human function curve. With special reference to cardiovascular disorders: part I. Practitioner. 1976; 217(1301): 765-770. PMid: 995833.

[2] West CP, Huschka MM, Novotny PJ, et al. Association of perceived medical errors with resident distress and empathy: a prospective longitudinal study. JAMA. 2006; 296(9): 1071-1078. PMid: 16954486. https://doi.org/10.1001/jama.296.9.1071

[3] Arora S, Aggarwal R, Moran A, et al. Mental practice: effective stress management training for novice surgeons. J Am Coll Surg. 2011; 212(2): 225-233. PMid: 21276534. https://doi.org/10 $.1016 / \mathrm{j} \cdot$ jamcollsurg . 2010.09.025

[4] Flinn JT, Miller A, Pyatka N, et al. The effect of stress on learning in surgical skill acquisition. Med Teach. 2015; 1-7.

[5] Shanafelt TD, Bradley KA, Wipf JE, et al. Burnout and self-reported

Published by Sciedu Press patient care in an internal medicine residency program. Ann Intern Med. 2002; 136(5): 358-367. PMid: 11874308. https://doi.or g/10.7326/0003-4819-136-5-200203050-00008

[6] Zhong X, Xiao Y, Huang R, et al. The effects of overnight sleep deprivation on cardiovascular autonomic modulation. Zhonghua Nei Ke Za Zhi. 2005; 44(8): 577-580. PMid: 16194407.

[7] Thomas C, Power C. Shift work and risk factors for cardiovascular disease: a study at age 45 years in the 1958 British birth cohort. Eur J Epidemiol. 2010; 25(5): 305-314. PMid: 20237824. https://doi.org/10.1007/s10654-010-9438-4

[8] Vrijkotte TG, van Doornen LJ, de Geus EJ. Effects of work stress on ambulatory blood pressure, heart rate, and heart rate variability. Hypertension. 2000; 35(4): 880-886. PMid: 10775555. https : //doi.org/10.1161/01.HYP.35.4.880

[9] Warburton DE, Nicol CW, Bredin SS. Health benefits of physical ac- 
tivity: the evidence. CMAJ. 2006; 174(6): 801-809. PMid: 16534088. https://doi.org/10.1503/cmaj.051351

[10] Mota MC, De-Souza DA, Rossato LT, et al. Dietary patterns, metabolic markers and subjective sleep measures in resident physicians. Chronobiol Int. 2013; 30(8): 1032-1041. PMid: 23964589. https://doi.org/10.3109/07420528.2013.796966

[11] Cecil J, McHale C, Hart J, et al. Behaviour and burnout in medical students. Med Educ Online. 2014; 19: 25209. PMid: 25160716. https://doi.org/10.3402/meo.v19.25209

[12] Bautmans I, Gorus E, Njemini R, et al. Handgrip performance in relation to self-perceived fatigue, physical functioning and circulating IL6 in elderly persons without inflammation. BMC Geriatr. 2007; 7: 5 . PMid: 17331228. https://doi.org/10.1186/1471-2318-7-5

[13] Song MH, Tokuda Y, Nakayama T, et al. Intraoperative heart rate variability of a cardiac surgeon himself in coronary artery bypass grafting surgery. Interact Cardiovasc Thorac Surg. 2009; 8(6): 639-641. PMid: 19286681. https://doi.org/10.1510/icvts.2008.195941

[14] Plews DJ, Laursen PB, Stanley J, et al. Training adaptation and heart rate variability in elite endurance athletes: opening the door to effective monitoring. Sports Med. 2013; 43(9): 773-781. PMid: 23852425. https://doi.org/10.1007/s40279-013-0071-8

[15] Eisenach JH, Sprung J, Clark MM, et al. The psychological and physiological effects of acute occupational stress in new anesthesiology residents: a pilot trial. Anesthesiology. 2014; 121(4): 878893. PMid: 25093592. https ://doi.org/10.1097/ALN . 000000 0000000397
[16] Hernández-Gaytan SI, Rothenberg SJ, Landsbergis P, et al. Job strain and heart rate variability in resident physicians within a general hospital. Am J Ind Med. 2013; 56(1): 38-48. PMid: 22886873. https://doi.org/10.1002/ajim. 22098

[17] Kuhn EW, Choi YH, Schönherr M, et al. Intraoperative stress in cardiac surgery: attendings versus residents. J Surg Res. 2013; 182(2): e43-49. PMid: 23228324. https://doi.org/10.1016/j.jss. 2012.11 .011

[18] Spielberger CD, Gorsuch RL, Lushene RE. Stai manual for the StateTrait Anxiety Inventory. [S.1.]: Consulting Psychologist Press; 1970.

[19] Phitayakorn R, Minehart RD, Hemingway MW, et al. Relationship between physiologic and psychological measures of autonomic activation in operating room teams during a simulated airway emergency. Am J Surg. 2015; 209(1): 86-92. PMid: 25454964. https : //doi.org/10.1016/j.amjsurg.2014.08.036

[20] Sargent MC, Sotile W, Sotile MO, et al. Quality of life during orthopaedic training and academic practice. Part 1: orthopaedic surgery residents and faculty. J Bone Joint Surg Am. 2009; 91(10): 23952405. PMid: 19797575. https://doi.org/10.2106/JBJS.H.0 0665

[21] Andreatta PB, Hillard M, Krain LP. The impact of stress factors in simulation-based laparoscopic training. Surgery. 2010; 147(5): 631-639. PMid: 20414972. https ://doi.org/10.1016/j.surg .2009 .10 .071 